\title{
Research on the buffering effect of rubber on the Non-Axial symmetry explosive loading
}

\author{
Ming Song ${ }^{\mathrm{a}}$ and Gaofeng Wei \\ School of Mechanical \& Automotive Engineering, Qilu University of Technology, Jinan, Shandong 250353, China
}

\begin{abstract}
The security shell of coal mine rescue capsule may be hit by the explosive in tunnel. We used the finite element software to established a model of security shell including three layers. The middle layer is made by rubber. Multiple simulation experiments on the buffering effect of rubber has been carried out. The buffering effect of rubber under the Non-Axial symmetry explosive loading is confirmed by the results. The rubber layer absorbs a part of the vibration energy on the outer layer. And it will transmit the last of energy to the inner layer. The rubber layer absorbs most of the small shock on the outer layer, too. The effect of rubber with different hardness and position has been discussed.
\end{abstract}

Keywords: rubber; explosive load; buffering effect.

\section{Introduction}

Rubber is a kind of high elastic polymer material[1]. It is widely used in industrial production and daily life due to the insulating property, waterproof property and easy processing. Rubber components are used from giant ships to Nano-mechanics, such as tire, damping spring, sealing ring and equipment shell[2,3]. The damping spring made by rubber can reduce the impact of vibration, which will protect the important machine. The rubber absorbs the vibrate energy from outside, and release it slowly. This process will reduce the destruction effect of vibration.

The rescue capsule is a coal mine safely equipment. The major function of it is protecting people from the disaster. In order to achieve the objectives, the security shell of it must protecting the equipment and trapped person from the explosion shock wave. Because the security shell facing the shock wave directly, the primary objective of security shell design is to ensure the integrity of the shell under shock wave. On the other hand, the vibration on the outer layer of security shell must been eliminated, which can destroy the equipment and hurt the person inside shell.

The rubber can be used on the security shell of the rescue capsule[4,5]. The middle layer of the security shell can be made by rubber. It will absorb the vibration on outer layer when the shock wave impact. It should also be noted that, the explosive loading in tunnel is non-axial symmetry. The subject on the buffering effect of rubber under the non-axial symmetry explosive loading need more in-depth study, which can increase the buffering effect of rubber to protecting the equipment and trapped person.

This paper proposes a new design method by using the rubber as a middle layer on security shell of the rescue capsule, in order to reducing the vibration on the inner layer under the shock wave. A

a Corresponding author : dears1007@163.com 
number of simulation experiments have been carried out to study the buffering effect of rubber under non-axial symmetry explosive loading. The effect of rubber with different hardness was also reviewed.

\section{Security shell simulation modeling}

The coal mine rescue capsule is made up of three parts: security shell, chassis and moving mechanism. The security shell is made by high pressure vessel steel using welding method. It is connected to the chassis and moving mechanism by bolts. The rescue capsule is placed in tunnel nearby the work place. The oxygen, food and water will be provided by support equipment in security shell.

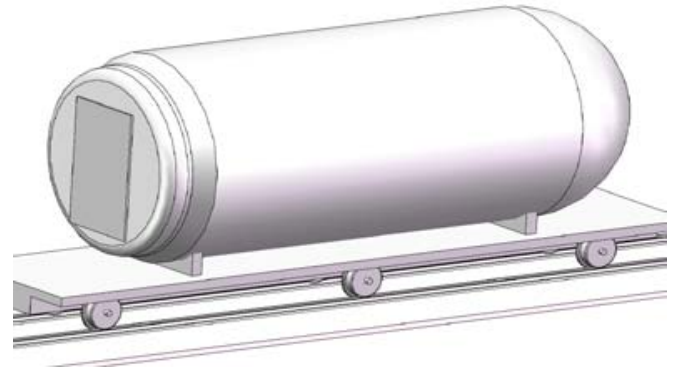

Figure 1. Cabin of the coal mine rescue capsule

The coal mine rescue capsule is shown in Fig. 1. It can be seen from Fig.1 that the security shell is composed of three parts: cylindrical shell, spherical shell and explosion proof door. The cylindrical shell on one end of the security shell will facing the shock wave, meanwhile, the explosion proof door will back toward it. In order to against the explosion shock wave and reduce the vibration of shell, the security shell is made up of three layers: inner layer, middle layer and outer layer. The outer and inner layers is made by steel. The middle layer is made by rubber. There is no direct contact between outer and inner layers. The outer and inner layers are both connected to the middle layer. All of the equipment inside the shell are set up on the inner layer. The chassis and moving mechanism will be ignored, because they have less volume and weight compared with the security shell. The explosion proof door will be ignored too. The security shell is simplified as an abstract model as shown in Fig. 2.

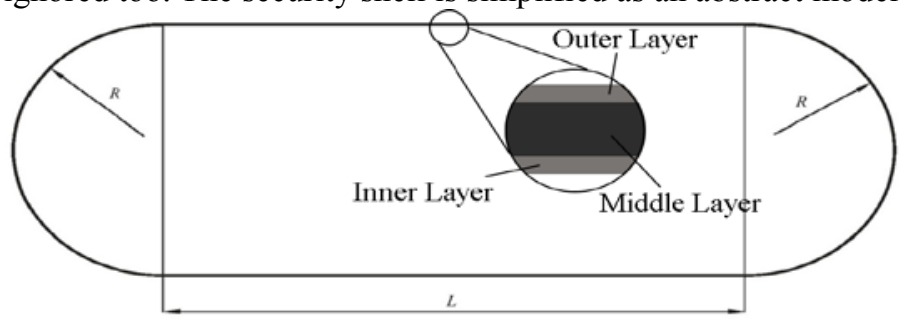

Figure 2. Parameters of security shell

The shell and environment model are built by finite element software. The model is shown in Fig. 3. In order to reduce the influence of environmental factors, the experiment tunnel is set as a cylinder space filling with ideal air at 20 degrees centigrade. The security shell is placed in it nearby the wall. The explosive source is simplified as a spherical TNT on one end of the tunnel, and be detonated at the beginning.

The security shell is meshed by Lagrange hexahedral element. The outer, inner and middle layers are meshed separately. The mesh cell is connected to another, using node superposition technology, between two layers. The outer and inner layers are defined as the DMATEP material, meanwhile, the middle layer using RUBBER1 define tab.

The Euler hexahedron grid is used for defining the cylindrical space. The spherical TNT is defined by the Jones-Wilkins-Leeequation of state equation[6,7]. In the model of security shell and tunnel, 
general coupling method is used to define the interface between the Lagrange and Euler units. The interaction between two kind of units is described by the interface using this method. But the node in Lagrange and Euler units don't need maintain the same coordinates, which can be used in more diverse environment.

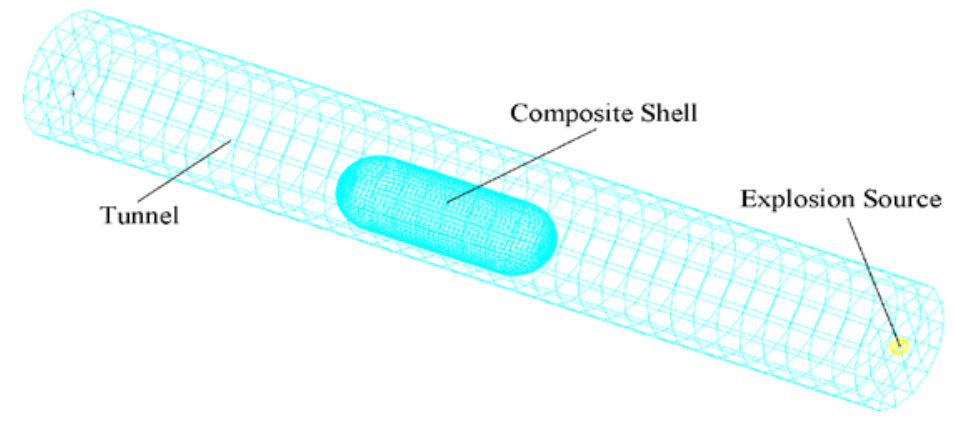

Figure 3. Shell and tunnel model

\section{Simulation result}

The material constants such as density, $\mathrm{C}_{1}$ and $\mathrm{C}_{2}$ need to be input when the middle layer is defined as the RUBBER1 material. The density of rubber will not change very much with the rubber composition. But the constants $\mathrm{C}_{1}$ and $\mathrm{C}_{2}$ will change obviously with the IRHD of the rubber, which will influence the buffering effect of rubber. So the constants $C_{1}$ and $C_{2}$ are set up based on some IRHD value. When IRHD is 40 , the $\mathrm{C}_{1}$ and $\mathrm{C}_{2}$ are set to $0.28 \mathrm{MPa}$ and $0.03 \mathrm{MPa}$. When IRHD is 60 , the $\mathrm{C}_{1}$ and $\mathrm{C}_{2}$ are set to $0.7 \mathrm{MPa}$ and $0.035 \mathrm{MPa}$. When IRHD is 70 , the $\mathrm{C}_{1}$ and $\mathrm{C}_{2}$ are set to $1.1 \mathrm{MPa}$ and $0.02 \mathrm{MPa}$. The shell is fixed with 8 fix points, which set on the outer layer of the shell. All of the fix points are divided into two groups. One group is set on the cylindrical shell nearby the facing-blast spherical shell, while the another group is nearby the second end of cylindrical shell. The angle between each points is 90 degrees in one group. Each fix points are defined as the 6-DOF constraint.

As the composite shell was fixed in the tunnel, the displacement of shell can't be used as the measure index. On the other hand, when shock wave hit the shell, the stress wave in shell will spread from one side of the shell to another, and shocking repeatedly. So the equivalent stress and stress wave are can't be used as the measure index, over a period of time. Finally, we set up some test point on the security shell, using the acceleration on them as the comparison object. We will compare the date between different test point position and rubber IRHD to revealing the buffering effect of rubber.

The stress distribution of inner layer under non-axial symmetry explosive loading at $0.05 \mathrm{~s}$ is shown in Fig. 4. In Fig. 4a-c, there is the stress distribution of fixed shell under different IRHD. In Fig. $4 \mathrm{~d}-\mathrm{f}$, there is the stress distribution of unfixed one. It can be seen from the picture that the area of stress doesn't has huge change. But the peak value of stress is increasing with the IRHD. It should be noted that, the fixed mode plays a great part in the stress distribution. Both of the peak value and area of stress are increasing when the shell is fixed.

The acceleration curve on the test point is shown in Fig. 5. The test point is set on the top of spherical shell facing the explosion shock wave with different IRHD, as shown in Fig. 5a-c. It can be seen from the picture that the outer and inner layers' movement trend are changed to different patterns by the rubber middle layer. There are many small shake on the acceleration curve collected from outer layer. It is clearly that the explosion shock wave causes a great vibration on the outer layer with those small shock, which will take a lot of damage to the inside equipment. However, the inner layer's acceleration curve tells a different story. The peak value of the acceleration is smaller than the outer layer. And, there is not obvious small shock on the curve. It is because the rubber middle layer absorbed a part of vibration energy on the outer layer. Then the rubber transmits the rest of energy to the inner layer. Rubber materials play a role in cushioning shock waves. It should be noted that the acceleration gap between outer and inner layers is falling with the increase of the IRHD. When IRHD 
is 70 , the acceleration curves on outer and inner layers are coincident. But the small shock is absorbed by the rubber middle layer too. In Fig. $5 \mathrm{~d}$, there is the acceleration curve on the test point in inner layer with different IRHD. It became further apparent that the peak value of acceleration is declining and the time of vibration start even later when IRHD is reduced. It means that the rubber can play a role in buffering effect not only reducing vibration amplitude but also delaying vibration time.

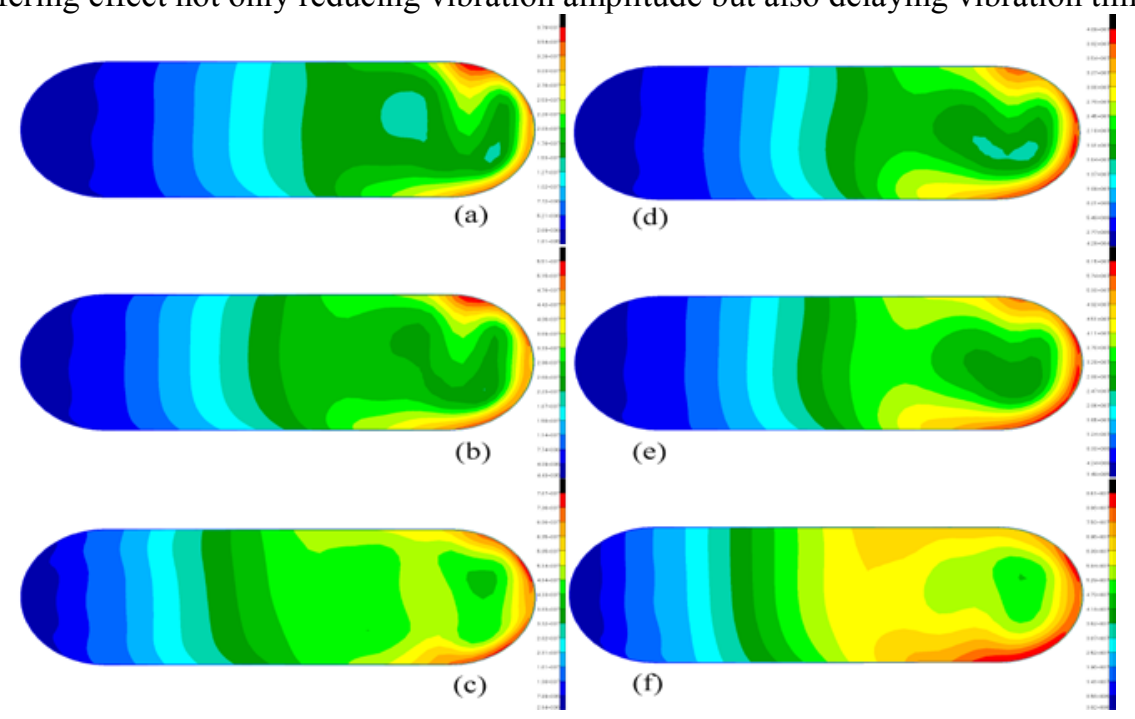

Figure 4. Simulation results of the inner layer at $0.05 \mathrm{~s}$

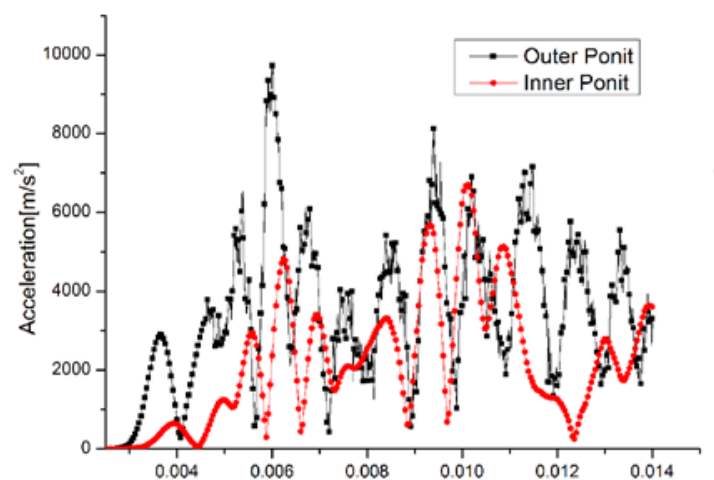

(a) Time[s]

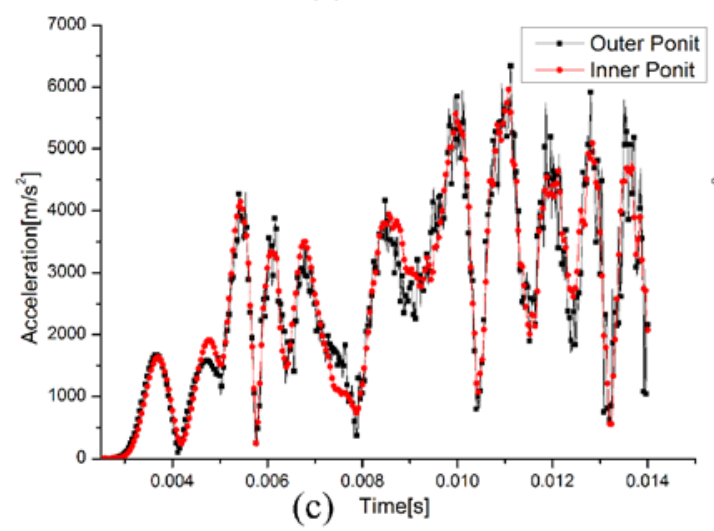

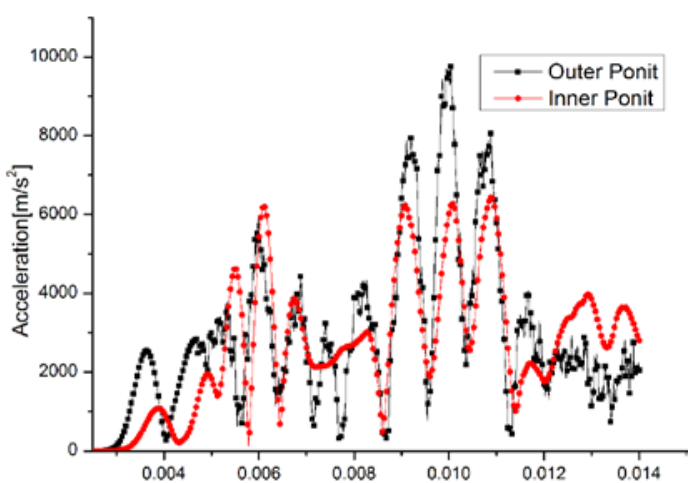

(b) Time[s]

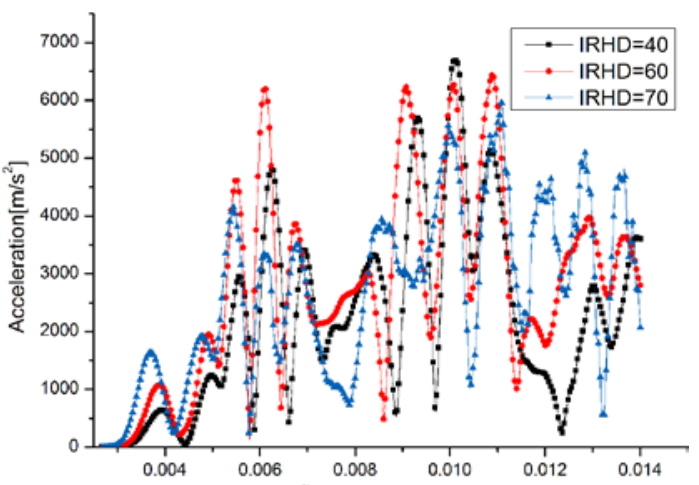

(d) Time[s]

Figure 5. Acceleration on the front test point 
The acceleration curve on other test points are shown in Fig. 6a-d. The position of test points is on the top, on the side, on the bottom of the cylindrical shell, and on the top of the back spherical shell. It can be seen from the picture that the buffering effect of rubber on shell at different position are not the same. The acceleration curve on the top of the cylindrical shell with different IRHD are basically similar, as shown in Fig. 6a. There are only two different points in the curve. The test result on the side point of the cylindrical shell is shown in Fig. $6 \mathrm{~b}$. There is no obvious difference among the different IRHD. It is interesting that the difference among different IRHD acceleration is larger than the former. The peak value of acceleration on the bottom of the shell has an obviously decrease when the IRHD reduced as shown in Fig. 6c. The situation is shown in Fig. 6d is more complex. When the IRHD is 70 the acceleration is larger than others, although the acceleration difference between 40 and 60 is not obvious. It should be noted that the time of vibration start even later when IRHD is reduced just like the situation in Fig. 5.

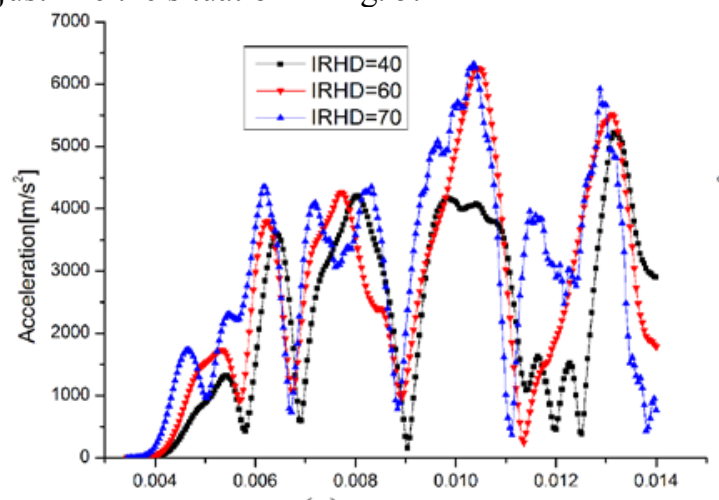

(a) Time[s]

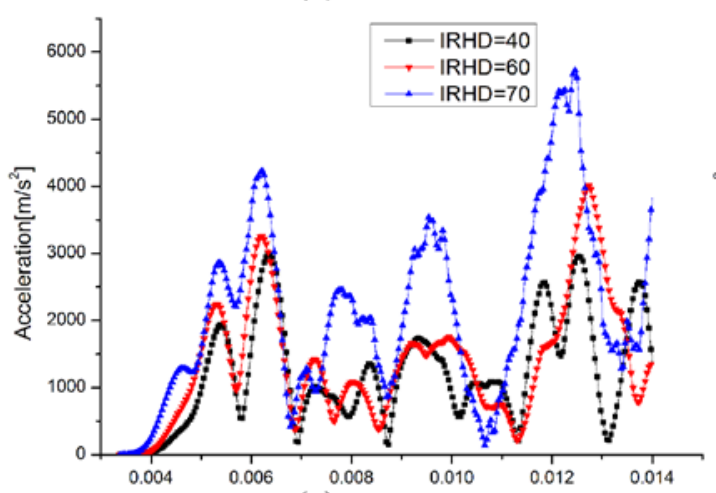

(c) Time[s]

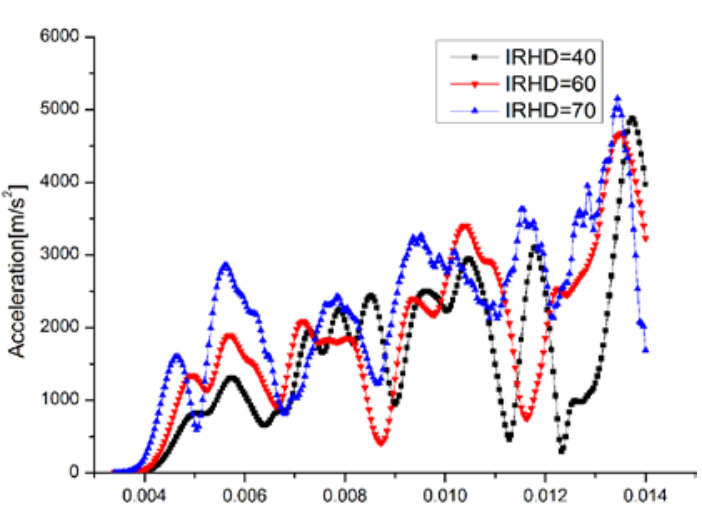

(b) Time[s]

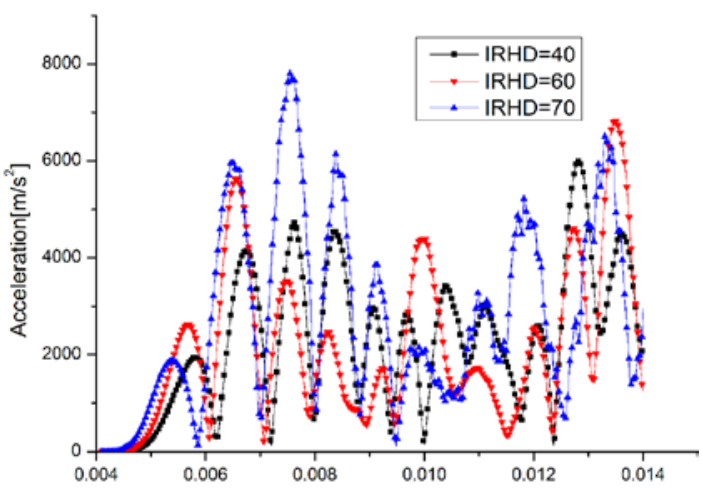

(d) Time[s]

Figure 6. Acceleration on other test points

\section{Summary}

In this paper, the buffering effect of rubber under the non-axial symmetry explosive loading is been researched by carrying out a number of simulation experiments. The impact of IRHD on the buffering effect of rubber is been reviewed. The buffering effect of rubber under the non-axial symmetry explosive loading is confirmed. The rubber middle layer can absorb the small shock in the vibration caused by the shock wave on the outer layer. The rubber middle layer can slow down the transmission speed of vibration. Generally speaking, the rubber middle layer with minor IRHD can absorb more vibration on the outer layer. But the buffering effect of IRHD between different position on the shell is not the same. 


\section{Acknowledgement}

This research was financially supported by the National Natural Science Foundation of China (11271234).

\section{References}

1. Borggreve, R. J. M., Gaymans, R. J., \& Eichenwald, H. M. Impact behaviour of nylon-rubber blends: 6. influence of structure on voiding processes; toughening mechanism. Polymer. 30 (1989) $78-83$.

2. G.Y. Wu, H.K. Jia, W. Zhou, X. Li, Simulation on anomalous response of spherical shell subjected to blast loading, J. Vib. Shock. 30 (2011) 93-96.

3. Liu, F., Chen, G., Li, L., \& Guo, Y. Study of impact performance of rubber reinforced concrete, Construction \& Building Materials. 36 (2012) 604-616.

4. Fatt, M. S. H., \& Sirivolu, D, Blast response of double curvature, composite sandwich shallow shells, Engineering Structures. 100 (2015) 696-706.

5. Sprenkle, W. E., \& Southern, J. H, Rubber-modified polystyrene impact as a function of flame-retardant additive solubilization, Journal of Applied Polymer Science. 26 (7) 2229-2238.

6. J.C. Xu, Y. Zhang, Nonlinear dynamic stability analysis of truncated sandwich shallow spherical shells subjected to explosive impacts, Eng. Mech. 28 (2003) 150-156.

7. Iluk, A., Modeling and evaluation of loads in vehicles subjected to mine blast, Solid State Phenomena. 220-221 (2015) 207-212. 